\title{
Production of Few-Layer Graphene in Synthetic Oil Using a Rod Drum Mill
}

\author{
G.B. Zhumagaliyeva ${ }^{1}$, V.F. Pershin ${ }^{2} *$ \\ ${ }^{1}$ Zhangir Khan West Kazakhstan Agrarian-Technical University, \\ 51, Zhangir Khan St., Uralsk, 090009, Republic of Kazakhstan; \\ ${ }^{2}$ Tambov State Technical University, 106, Sovetskaya St., Tambov, Russia \\ *Corresponding author. Tel.: +7 91065253 77. E-mail: pershin.home@mail.ru
}

\begin{abstract}
The article analyzes the methods of obtaining few-layer and multilayer graphene in machines where the main working body is a smooth rotating drum. Possible mechanisms for separating crystalline graphite particles are considered. The method of graphite exfoliation only due to shearing forces is viewed as the most economical one. A new design of the rod mill where only shearing forces affect graphite particles was introduced. The results of experimental studies on the influence of geometrical and mode parameters on the intensity of the shear exfoliation process are presented. The dependence for calculating the performance of a rod drum mill was obtained. A parametric series of mills with a capacity for few-layer and multilayer graphene from 0.077 to $0.884 \mathrm{~g} / \mathrm{h}$ was developed.
\end{abstract}

\section{Keywords}

Few-layer grapheme; shear exfoliation; light transmission coefficient; performance; parametric series.

(C) G.B. Zhumagaliyeva, V.F. Pershin, 2019

\section{Introduction}

Starting from the second half of the nineteenth century, grinding in ball drum mills has been widely used in various industries. Currently grinding using balls is one of the largest industries in the world [1]. A little later, planetary mills were invented, but the rotating drum and spherical grinding bodies also play the main roles in the grinding process. Moreover, since the gravitational force field acting on particles of material in traditional drum mills and the field of centrifugal forces acting on particles in planetary mills are potential fields, it can be assumed that the movement modes of the material and grinding bodies in drum and planetary mills are similar. The intensity and efficiency of grinding depends on the mode of movement of the grinding balls and material in the cross section of a rotating drum. The movement modes of the material in the cross section of the drum (Fig. 1) are avalanching, slumping, rolling, cascading, cataracting, and centrifuging. In practice, two modes are used more often than others, i.e. cascading [3, 4] and cataracting $[5,6]$. The cascade or circulation mode is usually used in mixers [7 - 10], granulators [11 - 14], classifiers or screens [15], cement kilns $[16,17]$, general issues of the bulk material movement in rotating drums are discussed in $[18,19]$. In the circulation mode of movement [20], the bulk material in the cross section of a smooth rotating drum forms a closed circulation loop. Part of the material particles rises up together with the rotating drum shell, forming a "rising layer", while another part of the particles rolls down forming a "rolling layer". Many researchers in the mathematical description of the material particles movement process make the assumption that the boundary between the rising and sliding layers is symmetrical about the circulation center. This assumption is correct only in the transition from a periodic collapse mode (slumping mode) to a circulation mode (cascading mode).

In fact, the specified boundary is clearly asymmetric, relative to the center of circulation (cascading mode, Fig. 2). 

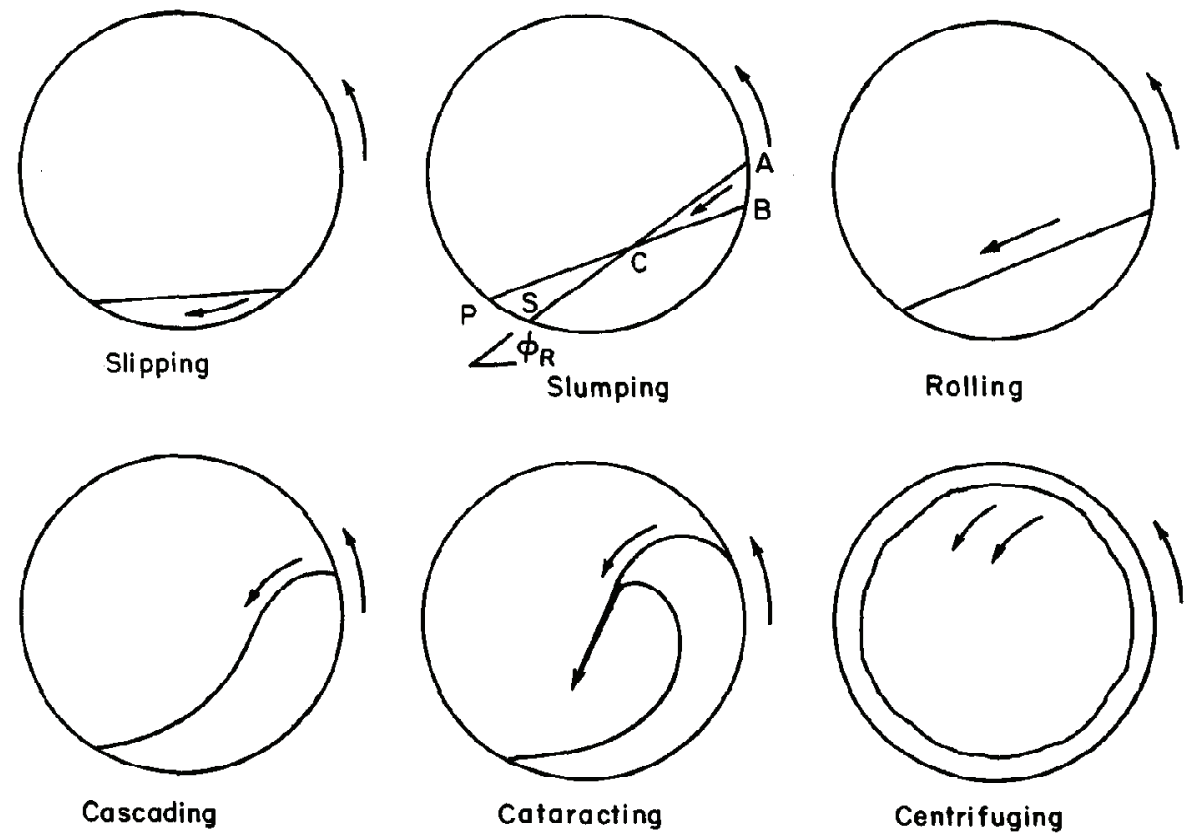

Fig. 1. Schematic diagram showing modes of transverse bed motion [2]

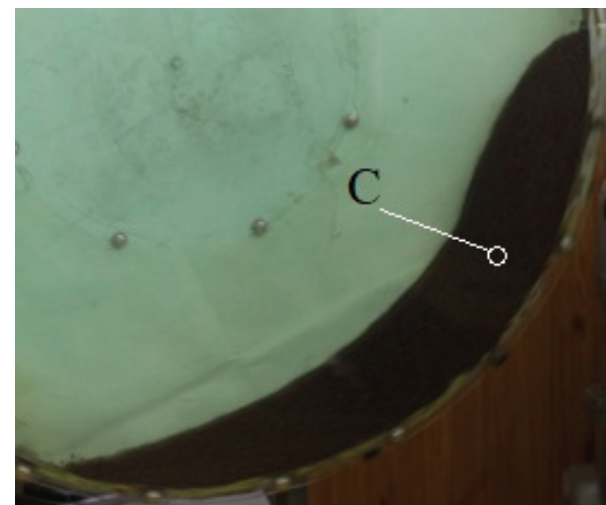

Fig. 2. The circulation mode of the bulk material movement

Different mathematical models are used to describe the bulk material movement in the cross section of a smooth rotating drum [21 - 24]. The main disadvantage of these models lies in the complexity of the experimental determination of numerous parameters. In [20], the energy approach adequately describes the distribution of the material and calculate the movement parameters of particles with a minimum of assumptions was proposed [25].

\section{Analysis of the grinding process in smooth rotating drums}

Machines, the main working body of which is a smooth rotating drum, are widely used for grinding different materials. First of all, these are ball drum mills and ball planetary mills.
Grinding building materials, such as gypsum, is used for thousands of years. In particular, gypsum was used in the construction of the pyramids, in about 3700 BC. Gypsum is found on all continents of the world and is one of the leaders among construction materials [26]. In this work, the effect of mode parameters on the intensity of gypsum grinding in drum ball mills was studied. Gypsum is widely used as special fillers, additional white pigment, fertilizer in agriculture, a binder of various building materials, as well as in dentistry. In all these industries, gypsum is needed in an ultra-fine ground form [27].

For a long time, the processes of grinding, in particular in ball mills, were subjected to statistical and kinetic analysis [28, 29], especially widely used ball mills in the mining industry [30]. The traditional forms of the grinding bodies are balls and rods [31]. Studying the behavior of the grinding load and the effect of the balls on the material particles allows us to determine the conditions in which the grinding bodies have the maximum effect on the material and ensure the maximum performance of the mill [32]. In [26], the effect of drum rotation speed, drum fill factor with grinding balls, ball size, material fill factor of a drum, particle size distribution of grinding balls and material particles, as well as the grinding time on the grinding intensity and grain size of the finished material was investigated. Studies were performed on a laboratory stainless steel ball mill with a drum diameter of $200 \mathrm{~mm}$ and a drum length of $200 \mathrm{~mm}$. The grinding bodies were stainless steel balls with a density 
of $8000 \mathrm{~kg} / \mathrm{m}^{3}$ and with three different diameters $(2,3$ and $4 \mathrm{~cm})$. Gypsum ore was used as a grinding material. Pre-ore was ground in a jaw crusher to a maximum size of $-2 \mathrm{~mm}$. Methods for describing particle size distributions, which take into account not only the width of the size distribution [33], but also the "slope" of the particle size distribution curve [34] are of particular interest in this study. The concepts "wide" and "narrow" or "steep" particle size distribution curve are used [35]. This approach is more informative than the traditional one. The rotation speed of the drum varied from 50 to $90 \%$ of the critical speed, which is equal to:

$$
\omega_{\mathrm{cr}}=\sqrt{g / R},
$$

where $g$ is the acceleration of gravity, $9.81 \mathrm{~m} / \mathrm{s}^{2}$, $R$ is the inner radius of the drum, $\mathrm{m}$.

Experimental results showed that the most intensive grinding occurs at speeds of the drum rotation from 80 to $90 \%$ of the critical speed. Since at a speed of $80 \%$ of the critical speed, the energy consumption is substantially less, it is this speed that the authors recommend for industrial production.

In the past two decades, more adequate models have been developed using the finite element method, describing the movement and configuration of the loading circuit of grinding bodies in drums. However, finite element methods currently do not imitate the actual behavior of the particles to be ground and the effect of the particles on the balls movement; therefore, the true results of grinding are not predictable. The authors of [36] distinguish three main zones in the cross section of the drum: the grinding zone, where the balls collide with each other and crush the particles that fall into the collision zone; the ascending zone where the balls move up along the wall of the mill; the drop zone where the balls are in free fall. In fact, grinding occurs only in one zone. It is noted that if the material is small, the particles occupy the free space between the grinding balls. The balls fall on the balls located at the bottom and the grinding does not occur, because there are no material particles in the area where the balls hit. The paper presents the results of comparing calculations with experimental data obtained by other researchers. The influence of different parameters on the grinding intensity is analyzed. Despite the importance of this work for the theory and practice of grinding, it does not provide specific recommendations. Analyzing the influence of the balls diameter on the grinding process, it is noted that with an increase in the diameter, the impact energy increases, but at the same time the number of areas of simultaneous collision of the balls decreases. As the degree of the drum filling with grinding balls increases, the number of grinding zones increases, but at the same time the impact energy decreases, as the height of the falling balls decreases.

In conclusion, it should be noted that a grinding theory has been developed, which is based on the dispersion coefficient and the Peclet number in a continuous ball mill. The dispersion coefficient was expressed by statistical parameters taking into account the random walk of balls and particles. Then the statistical parameters were determined taking into account the geometrical and operational parameters of the mill. The obtained equations showed that the dispersion coefficient is a function of the dispersion zone size and the mobility of the balls in the grinding zone. The mobility is characterized by an average speed and free path. The results obtained from the theory agree with the experiment. In addition, the theory predicted a significant effect of the length and diameter of the mill, not only on productivity, but also on the quality of grinding. The results of this work are very useful in designing drum mills.

\section{Obtaining graphene structures in planetary mills}

After the discovery of the electric field effect in graphene in 2004 [37], the academic community was very interested in this material. Graphene has great potential for use in many areas due to its excellent thermal conductivity [38, 39]. Much effort was made to obtain graphene with one or several layers, including micromechanical separation of highly ordered pyrolytic graphite oxide [40], chemical vapor deposition [41, 42], separation of the graphite liquid phase [43 - 45], epitaxial growth [46], thermal peeling [47]. In [48], to obtain graphene structures, hexagonal graphite (HG) powders were used as a raw material. The HG powders were dried at $75^{\circ} \mathrm{C}$ for 2 hours in an oven. Then the dehydrated HG powders were ground in a Fritsch Pulverisette Premium line 7 laboratory planetary mill using balls with a diameter of $19 \mathrm{~mm}$ at $400 \mathrm{rpm}$ for 50 hours. The powders obtained after the grinding process were mixed with saturated acids, $\mathrm{H}_{2} \mathrm{SO}_{4}$ and $\mathrm{HNO}_{3}$, for 12 hours using a magnetic stirrer. The powder mixture filtered from the acidic mixture was irrigated with distilled water until its $\mathrm{pH}$ was neutral. The powder obtained at the end of this process is called graphite intercalating compound (GIC). GIC was heated to $950{ }^{\circ} \mathrm{C}$ to obtain expanded graphite (EG). EG is a structure in which the distance between the layers is increased compared to the original graphite, but the layers are still connected to each other by pecder-Waals bonds. EG was mixed in $\mathrm{N}$, $\mathrm{N}$-dimethylformamide (DMF) using an ultrasonic 
homogenizer (Bandelin Sonoplus, model HD 3200, $200 \mathrm{~W}$, at $50 \%$ power, i.e. $100 \mathrm{~W}$ ) for 2 hours. Next, the EG suspension in DMF with a concentration of $0.05 \mathrm{mg} / \mathrm{ml}$ was prepared. Then the mixture was centrifuged at $5000 \mathrm{rpm}$ for 8 hours to remove DMF. The powders were sprinkled with ethanol and dried in a vacuum oven at $75{ }^{\circ} \mathrm{C}$ for 4 hours to remove alcohol. The results of studies using high-resolution transmission electron microscopy (HRTEM, JEOL Jem 2100F), Raman spectroscopy (Witecsnom-Raman) using $532 \mathrm{~nm}$ laser excitation through a 100-fold objective and thermogravimetric analysis (TGA) of the samples showed that most of graphite has passed into an amorphous state as a result of strong shock influences. Fig. 3 shows TEM images of the resulting graphene sheets.

The photographs show graphene nanoplates found in each product area obtained as a result of the liquid- phase exfoliation method in a spherical planetary mill. Fig. $3 a$ and $3 b$ display a lot of sheets with several nanometers thickness. The width of these sheets reaches several micrometers. Electron diffraction (ED), shown in Fig. 3d, confirmed the crystallinity of the graphene structure. However, when the ED pattern was examined, it could also be argued that there were disordered areas in the structure. The inner parts of the layers and even partially the entire layer had disordered structures. The dark colored particles were present in graphene layers or / and areas close to them in all TEM images shown in Fig. 3. These particles were amorphous carbon structures. It was previously stated that partial amorphization occurs during HG grinding. $\mathrm{X}$-ray diffraction analysis carried out at the end of the grinding process also showed that amorphization was present in HG.

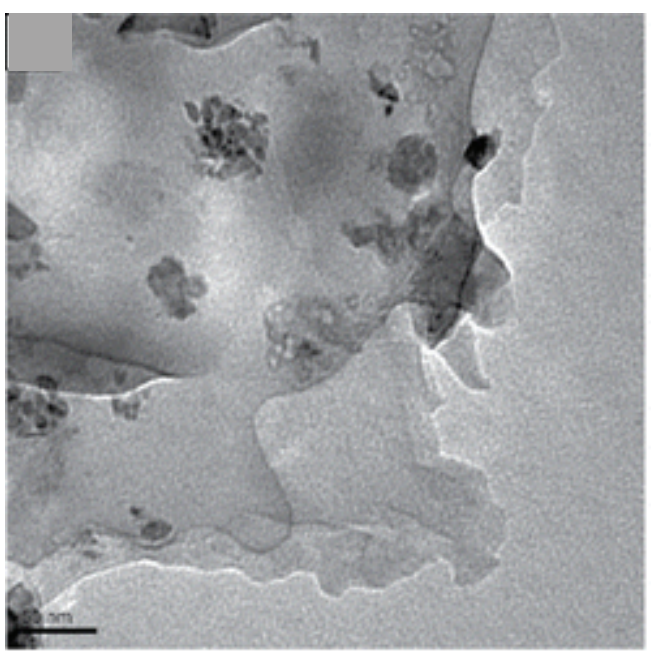

a)

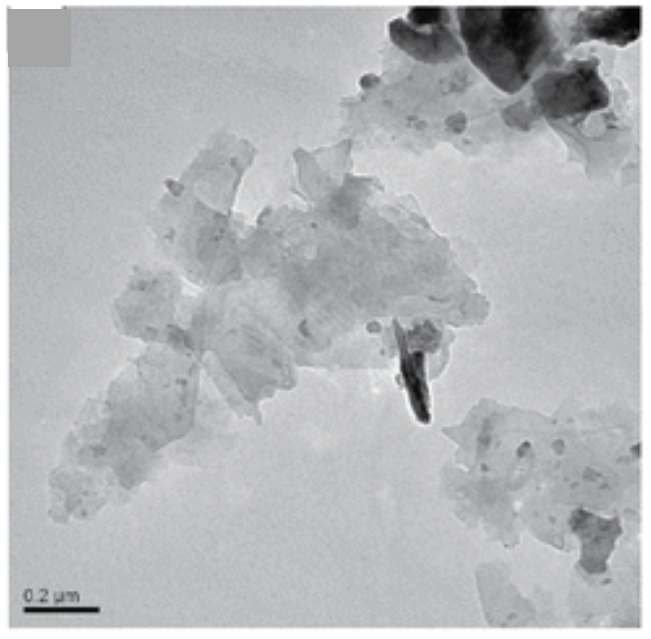

c)

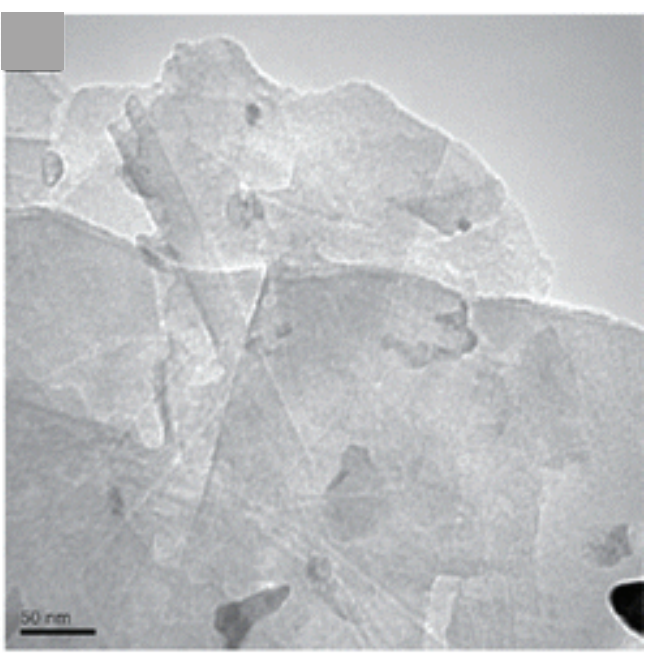

b)

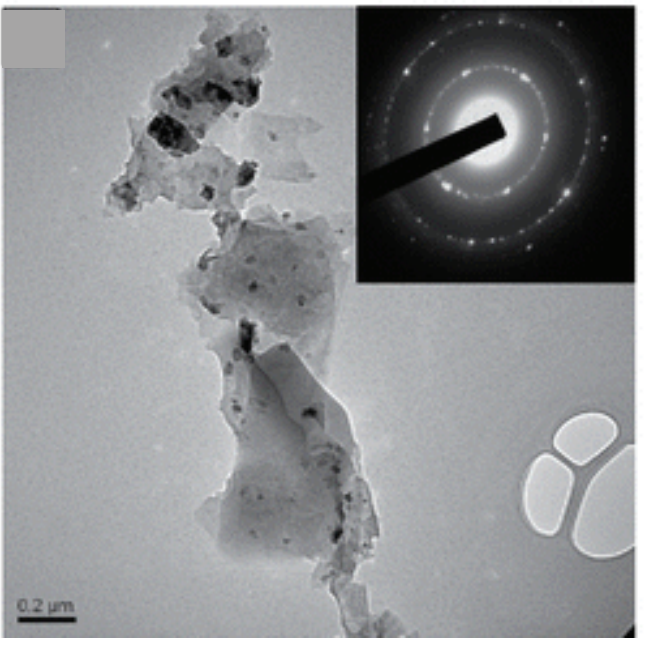

d)

Fig. 3. TEM micrographs $(a-c)$; Electron diffraction pattern of synthesized pectros layers $(d)$ [48] 


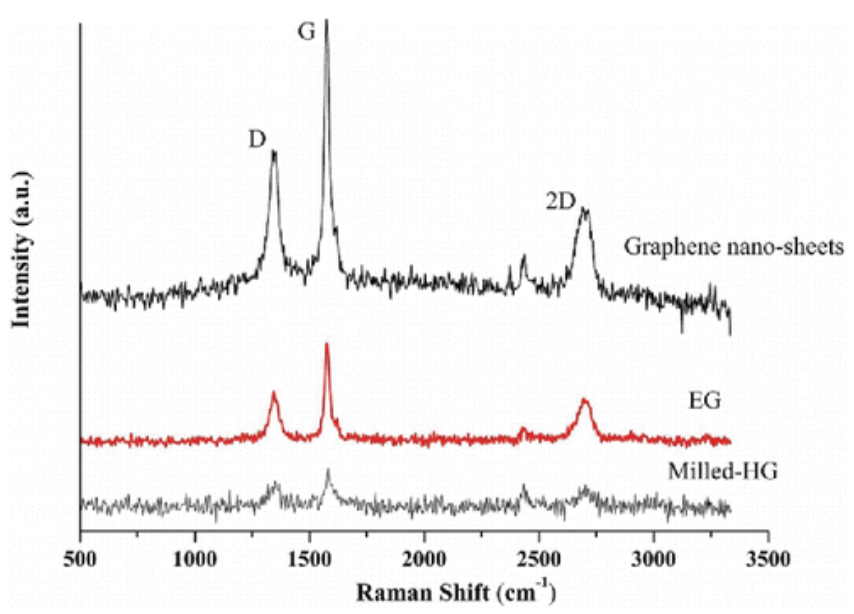

Fig. 4. Raman spectroscopy analysis of milled HG, EG, graphene [48]

The Raman spectroscopy is an effective method used to characterize carbon materials, both with an ordered and disordered crystalline structure. In addition, this method allows us to analyze the thickness of graphene nanoplastics. Fig. 4 shows the Raman spectra of HG, EG and synthesized graphene milled layers.

Fig. 3 presents characteristic peaks found in the Raman spectra of carbon materials. Among these peaks, the peak of the "D" band was observed at about $1350 \mathrm{~cm}^{-1}$. This peak was an indicator of defects found within the structure. A peak that is visible at about $1600 \mathrm{~cm}^{-1}$ is the peak of the "G" band. Carbon atoms linked by the sp2 bond are marked at this peak. In other words, it was a graphite array indicator. The " $2 \mathrm{D}$ " peak is observed at approximately $2700 \mathrm{~cm}^{-1}$. It is this peak that is used to determine the number of layers in multilayer structures. The intensity of the characteristic peaks in the Raman spectra of a sample obtained after 50-hour HG grinding is relatively low (Fig. 4). This situation indicates that amorphous carbon prevailed throughout the structure at the end of the 50-hour grinding.

In [49], high productivity was achieved in the production of few-layer graphene $(\leq 5$ layers $)$ by treating wood graphite in a planetary ball mill in the presence of dry ice for 48 hours. The resulting product has a high dispersibility in various solvents. The findings confirm the possibility of obtaining graphene as a result of shear effects on graphite without oxidizing the original graphite. In our opinion, one of the most promising areas of graphene production on an industrial scale is liquid-phase shear exfoliation in a drum mill, especially since there are examples of how to obtain nanoscale particles in ball drum mills [50].

\section{Graphite exfoliation shift mechanism}

Each crystalline graphite particle is a deck in which the cards are graphene sheets one carbon thick. The number of graphene sheets is different and can vary from hundreds to several thousand. If the reduction of graphene layers in graphite particles is considered as the final result of the exfoliation process, then it can be stratified only in two ways: tear one particle from another or move one particle relative to another. In the work [51] on the basis of molecular modeling, the delamination scheme shown in Fig. 5 was proposed. It should be noted that the substrate is taken only for clarity and instead you can use the second graphene sheet.

For this diagram of force and delamination variants, according to the laws of classical mechanics, the minimum force at which delamination can occur corresponds to the shear mechanism. The authors introduced the slip coefficient $\lambda$, the numerical value of which is determined by the following expression:

$$
\lambda=l /(1 l+1 d) .
$$

At $\lambda=1$, lateral sliding is absent $(d=0)$ and only vertical peeling of the $2 \mathrm{D}$ material occurs; at $\lambda=0$ $(\alpha=0)$, purely lateral sliding without vertical delamination occurs. Fig. 6 shows the action zones of the separation and shear mechanisms ( $b$ is the width of the graphene plate).

Thus, it is necessary to create a structure in which only shearing forces will be transmitted to the particle, without any effort of separation.

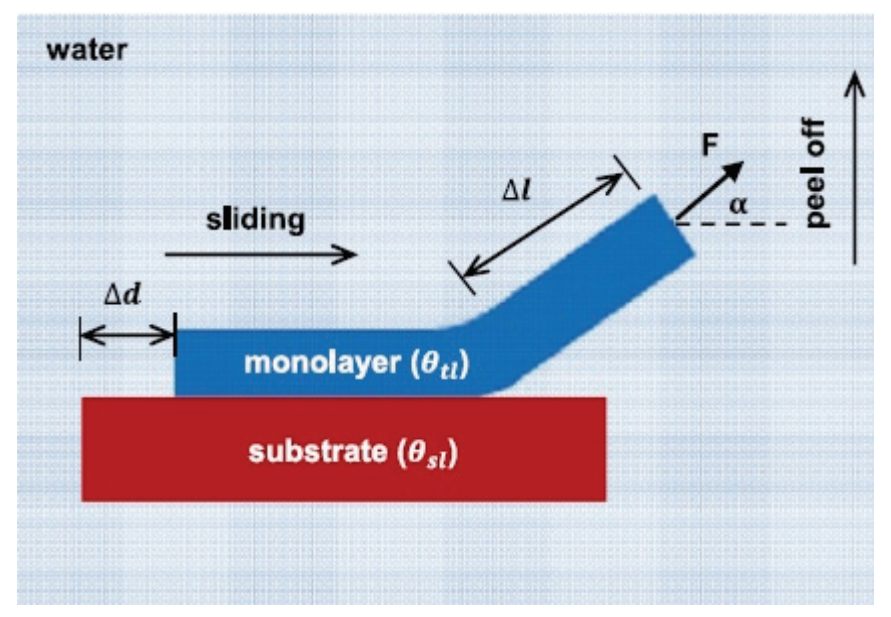

Fig. 5. Diagram of force when peeling the graphene layer from the substrate [51] 


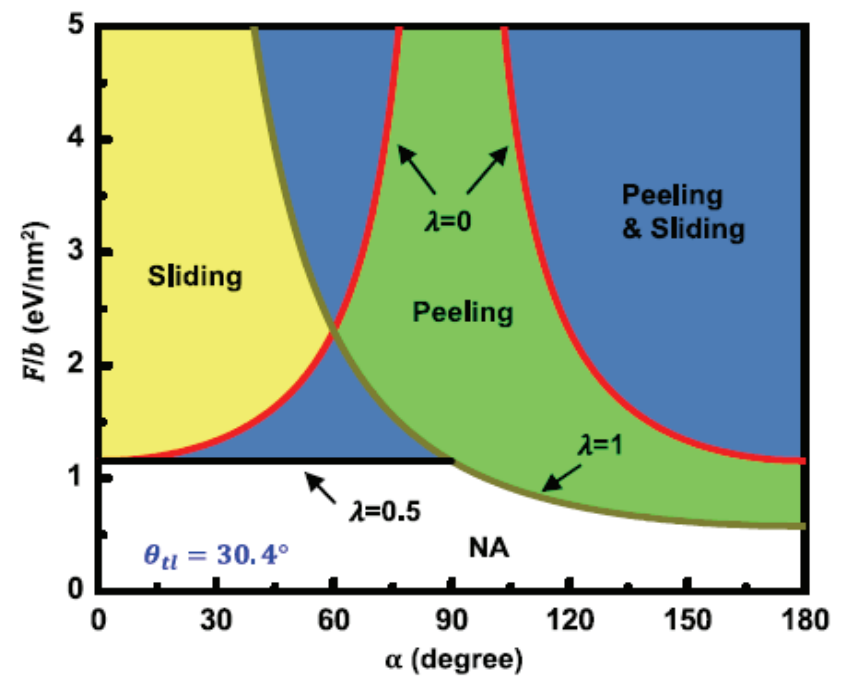

Fig. 6. Action zones of the separation and shear mechanisms [51]

\section{Rod drum mill for graphite liquid-phase shift exfoliation}

Fig. 1 shows that in a drum mill it is possible to organize a mode in which material periodically slides along the inner surface of the drum (slumping mode). The ability to organize such a mode for grinding balls was theoretically proved in [52], and experimentally confirmed in [53]. This paper establishes that some balls moving downward rotate without sliding relative to the inner surface of the drum. Initially, the production of graphene structures in the graphite grease was implemented in a laboratory planetary mill made in accordance with [54]. The study of the tribological characteristics of the graphite grease processed in a new design of the planetary mill showed that due to the formation of graphene structures all the characteristics have improved. In particular, the friction coefficient decreased by $1.5-2$ times [55]. It was also experimentally established that graphene increases the viscosity of the dispersion medium [56], therefore, when preparing greases, less thickener is required. Thus, the use of graphene is one of the promising methods for the commercial production of environmentally friendly grease [57].

Studies have been conducted on the replacement of grinding balls with rods, which made it possible to significantly increase the contact zone of the grinding bodies with the drum, i.e. increase the area of the zone in which the separation of graphite particles is possible. Taking into account the above circumstances, a new design of a rod drum mill [58] was developed to obtain graphene suspensions in an oil base. The principal difference of this design is that the rods are interconnected by a flexible cable, which eliminates the rotation of the rods in a rotating drum. Thus, the rods slide relative to the inner surface of the drum and on the particles that fall into the contact zone between the rods and the drum. Only shear forces are applied.

\section{Experiment, findings and discussion}

To obtain graphene suspensions, a laboratory drum mill with grinding rods was used, which were interconnected, as shown in Fig. 7. Video recording of the rods movement in the cross section of a smooth rotating drum showed that the rods interconnected by a flexible cable, as well as the free rods, periodically move upwards together with the drum and then slide down to the starting position. Shift effects on particles can occur only when the rods slide down. It was established experimentally that the time of the rods upward movement and the time of the rods sliding down are almost the same. Thus, during the time (T) when the material and rods stay in the rotating drum, the graphite particles are stratified during the time $\left(T_{E}\right)$ which is at best half the time $T$. To eliminate this drawback, the rods were connected to the fixed base using a bracket. Similarly, rods were fixed in a laboratory setup with a drum diameter of $140 \mathrm{~mm}$ and a length of $100 \mathrm{~mm}$. During the experiments, the angular velocity of the drum rotation was changed from 56 to $141 \mathrm{rpm}$, which is from 0.5 to 1.25 from $\omega_{\mathrm{cr}}$, but remained constant throughout one experiment.

Steel rods with a diameter of 8,10 and $12 \mathrm{~mm}$ were used. Studies were performed as follows. The initial suspension by mixing synthetic oil $5 \mathrm{~W} 40$ and graphite powder GSM-2 was prepared. The graphite concentration $\left(C_{O}\right)$ was changed from 5 to $20 \mathrm{wt}$. \%. The suspension was loaded into the drum where there was a certain number of rods with a certain diameter. The number of rods (interconnected) was changed from 4 to 12 . Every 3 hours the drum rotation was turned off and 3 samples were taken for analysis. The intensity of graphene structures formation was estimated from the change in the transmittance coefficient $K$, the numerical value of which characterizes the average integral number of layers in graphite particles [59]. This type of analysis requires a small amount of samples, which is very important for laboratory research. Fig. 8 shows the characteristic dependences of the coefficient $K$ on time. In this case, for dependence 1: the angular velocity of the drum rotation $(\omega)$ was equal to $11.8 \mathrm{~s}^{-1}\left(1.0 \omega_{\mathrm{cr}}\right)$, for dependence 2: $\omega=5.9 \mathrm{~s}^{-1}\left(0.5 \omega_{\mathrm{cr}}\right)$, for dependence 3: $\omega=14.2 \mathrm{~s}^{-1}\left(1.2 \omega_{\mathrm{cr}}\right)$, and the graphite concentration in the initial suspension $C_{O}=10 \mathrm{wt} . \%$ for all variants. 


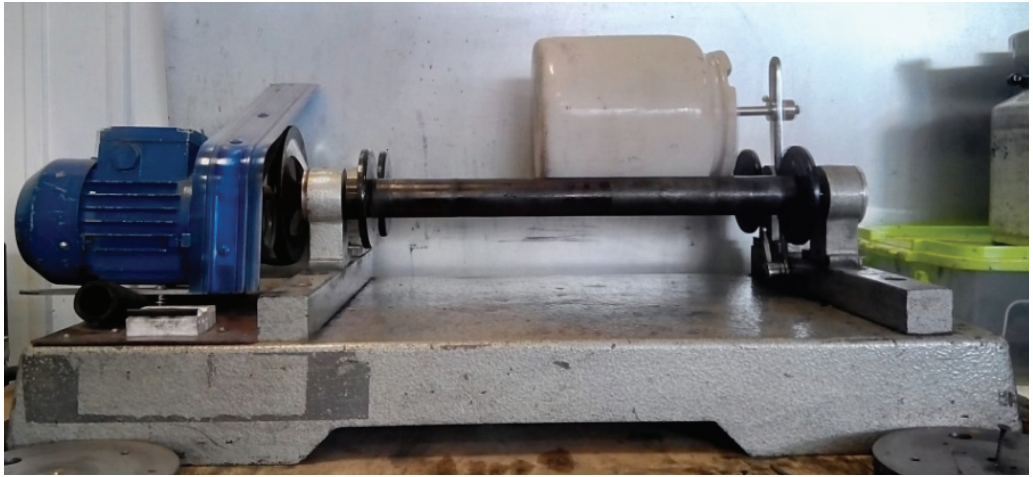

a)

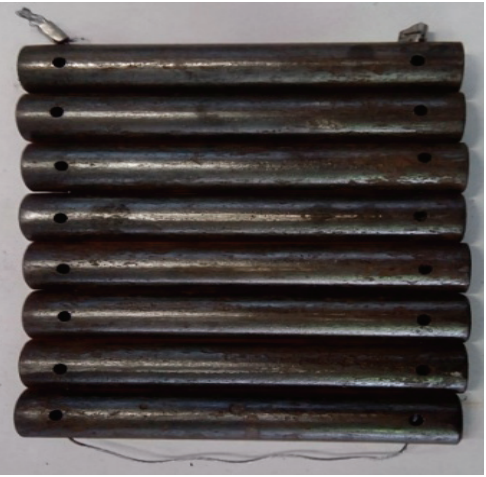

b)

Fig. 7 Experimental setup:

$a$-drum mill; $b$ - grinding rod load

The graph shows that at the beginning of the process the dependences can be considered linear, and the intensity of the process increases in direct proportion to the increase in the rotational speed. Fig. 9 presents the characteristic dependences of the coefficient $K$ on time, with different graphite concentrations in the initial suspension. The rotation speed of the drum was $11.8 \mathrm{~s}^{-1}$, but for dependence 1 : $C_{O}=10 \mathrm{wt} . \%$, and for dependence 2: $C_{O}=5 \mathrm{wt} . \%$. The graph displays that the obtained dependencies are similar to those shown in Fig. 9, i.e. at the beginning of the process the dependencies can be considered linear, and the intensity of the process increases in direct proportion to the increase in the graphite concentration in the initial suspension. When a certain concentration of few-layer and multilayer graphene, about 6-7 $\mathrm{mg} / \mathrm{ml}$, is reached in suspension, the intensity drops sharply and asymtotically approaches its limiting value. In obtaining the dependences that are necessary to predict the intensity of the liquid-phase shear exfoliation process, the initial period of the process is of interest. As the analysis of numerous experiments has shown, with various combinations of mode and geometric parameters, the time during which the intensity drops and the content of graphene structures in the suspension reaches the limiting values is no more than $20 \%$ of the total processing time of the initial suspension. It was also found that the optimal number of rods is one-fourth of the drum circumference located in the lower quadrant in the direction of the drum rotation. The pronounced dependence of the process intensity on the diameter of the rods during the experiments was not revealed. Perhaps this issue will be resolved when conducting research on a drum with a substantially large radius.

First of all increasing speed of the drum rotation of, leads to increasing the path that the graphite particles go through being in the contact zone of the rods with the drum inner surface. Therefore, it is logical

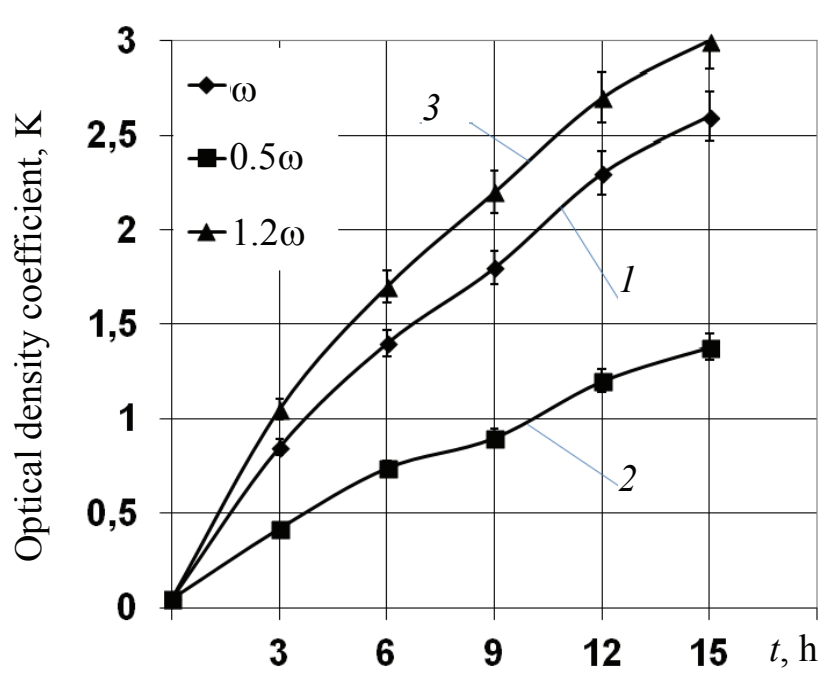

Fig. 8. Dependence of the optical density coefficient on time

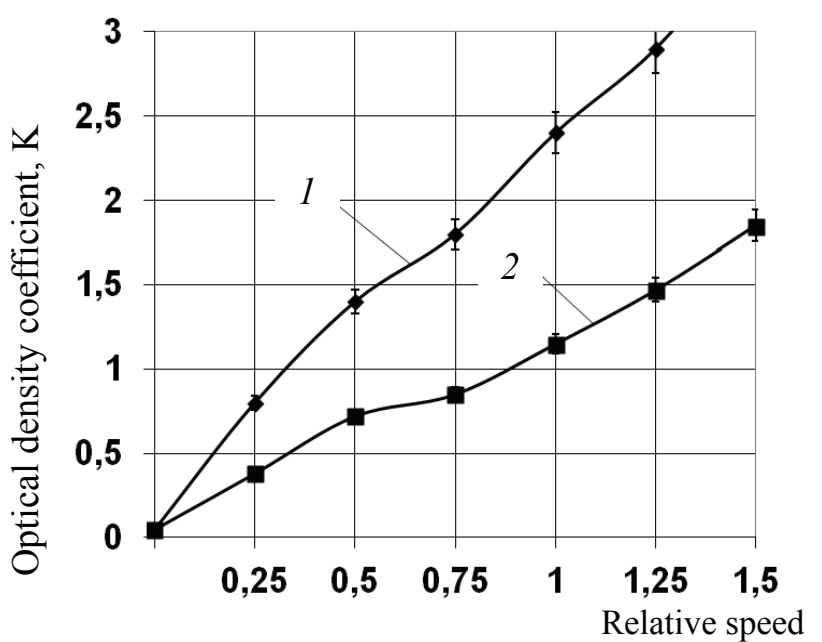

of rotation of the drum, $\omega / \omega_{\mathrm{CR}}$,

Fig. 9. Dependence of the optical density coefficient on the relative speed of the drum rotation 
to assume that the intensity of the process will be directly proportional to the radius of the drum. Taking into account the above justifications, the intensity of the exfoliation process and, ultimately, the performance of the rod mill $(Q)$ can be represented as follows:

$$
Q / Q_{L}=k C_{L}\left[\left(R / R_{L}\right)\left(L / L_{L}\right)\left(C_{O} / C_{O L}\right)\left(\omega / \omega_{L}\right)\right],
$$

where $k$ is a dimensionless coefficient of proportionality, the numerical value of which depends on the characteristics of the oil base.

The influence of the coefficient of filling the drum with a suspension, as a result of the experiments performed, was not revealed. The coefficient of filling the drum with suspension $(\xi)$ is numerically equal to:

$$
\xi=V_{S} / V_{D},
$$

where $V_{S}$ is the volume of the suspension, $V_{D}$ is the volume of the drum.

As $\xi$ increases, the volume of the suspension in the drum increases, but the number of particles in the shear zone does not change. Basis on this, with respect to the total volume of the suspension, an increase in the fewlayer and multilayer graphene concentration per time unit will decrease in direct proportion to the increase in $\xi$. However, the absolute value of the mass of these particles, and hence the performance, does not depend on $\xi$.

The dependence (3) includes all the main geometrical and mode parameters that characterize the mill and the exfoliation process. To test the possibility of using this dependence when designing rod mills, the numerical value of $Q_{L}$ was experimentally determined with the following parameters of the laboratory mill: radius $R=70 \mathrm{~mm} ; L=100 \mathrm{~mm} ; C_{O L}=10$ wt. \%; $\omega_{L}=\omega_{\mathrm{cr}} ; \quad \xi_{L}=0.1$. As a result of processing the experimental data, a value of $Q_{L}=0.064 \mathrm{~g} / \mathrm{h}$ was obtained, with few-layer and multilayer graphene concentration in suspension. After centrifugation and removal of sediment $C_{L}=6.4$ wt. $\%$ and light transmission coefficient $K=2.6$.

The verification of the adequacy of the dependence (3) was checked by comparing the calculation results with the experimental data. Experimental values $Q$ were determined in laboratory setups with the drum diameters of 140 and $200 \mathrm{~mm}$ for the following combinations of parameters:

$1-R=70 \mathrm{~mm} ; L=100 \mathrm{~mm} ; C_{O L}=5$ wt. $\%$; $\omega_{L}=0.5\left(\omega_{\mathrm{cr}}\right) ; \xi_{L}=0.13$;

$2-R=70 \mathrm{~mm} ; L=100 \mathrm{~mm} ; C_{O L}=10$ wt. \%; $\omega_{L}=0,5\left(\omega_{\mathrm{cr}}\right) ; \xi_{L}=0.1$;
$3-R=70 \mathrm{~mm} ; L=100 \mathrm{~mm} ; C_{O L}=10$ wt. $\%$; $\omega_{L}=\left(\omega_{\mathrm{cr}}\right) ; \xi_{L}=0.13$;

$4-R=70 \mathrm{~mm} ; L=100 \mathrm{~mm} ; C_{O L}=10$ wt. \%; $\omega_{L}=1.25\left(\omega_{\mathrm{cr}}\right) ; \xi_{L}=0.13$;

$5-R=100 \mathrm{~mm} ; L=120 \mathrm{~mm} ; C_{O L}=5$ wt. \%; $\omega_{L}=0.5\left(\omega_{\mathrm{cr}}\right) ; \xi_{L}=0.13$;

$6-R=100 \mathrm{~mm} ; L=120 \mathrm{~mm} ; C_{O L}=10$ wt. \%; $\omega_{L}=0.5\left(\omega_{\mathrm{cr}}\right) ; \xi_{L}=0.1$;

$7-R=100 \mathrm{~mm} ; L=120 \mathrm{~mm} ; C_{O L}=10$ wt. $\%$; $\omega_{L}=\left(\omega_{\mathrm{cr}}\right) ; \xi_{L}=0.13$;

$8-R=100 \mathrm{~mm} ; L=120 \mathrm{~mm} ; C_{O L}=10$ wt. $\%$; $\omega_{L}=1.25\left(\omega_{\mathrm{cr}}\right) ; \xi_{L}=0.13$;

$9-R=100 \mathrm{~mm} ; L=120 \mathrm{~mm} ; C_{O L}=20 \mathrm{wt} . \%$; $\omega_{L}=\left(\omega_{\mathrm{cr}}\right) ; \xi_{L}=0.13$;

$10-R=100 \mathrm{~mm} ; L=120 \mathrm{~mm} ; C_{O L}=20$ wt. \%; $\omega_{L}=1.25\left(\omega_{\mathrm{cr}}\right) ; \xi_{L}=0.13$.

The analysis of the obtained results showed that the deviations of the calculated productivity values from experimental values do not exceed $15 \%$. In our opinion, this is quite acceptable because finally a number of parameters, which ensure maximum performance and the required quality of the finished product, are determined during the commissioning tests of the industrial mill. These parameters include: $C_{O} ; \omega$; $\xi_{L}$. Based on these circumstances, we recommend, when designing rod mills, to provide the possibility of changing the angular velocity of rotation by about $20-30 \%$ of the calculated values.

Dependence (3) became the basis for the development of a methodology for calculating rod mills for producing few-layer and multilayer graphene in liquids. As the initial data, the performance of the mill and the concentration of graphene structures in the finished product are specified. On the basis of the specified parameters using the data bank obtained experimentally, the geometric and operating parameters are calculated.

Using this technique, a parametric series of rod mills with graphene productivity was developed in suspension containing at least 6.4 wt. \% graphene: $0.085 ; 0.269 ; 0.884 \mathrm{~g} / \mathrm{h}$. The modernization variants of serially produced laboratory mills $\mathrm{MSF} / 2, \mathrm{MSF} / 4$, MSF/8 and MSF/12 (Techno-Center LLC, Rybinsk, Yaroslavl Region) were developed. The numbers in the models indicate the volume of the porcelain drum in liters. A draft design of a modified mill with a unified bracket for fixing rods was prepared. If we consider the drums with dimensions which are actually used in industry, the performance of even a very small drum with a diameter of $1.4 \mathrm{~m}$ and a length of $1.5 \mathrm{~m}$ in terms of dry graphene structures is $370 \mathrm{~g} / \mathrm{h}$. 


\section{Conclusion}

The literature data analysis showed that it is possible to organize the mode of periodic slips of the grinding bodies in the rotating drum relative to the inner surface of the rotating drum. It is during the sliding of the grinding bodies that the separation of graphite particles and the formation of graphene structures occur. A new design of a drum mill was developed, in which the grinding rods constantly slide along the surface of the drum. Studies have been carried out on the process of liquid-phase shear exfoliation of graphite in oil, and it has been established that the concentration of few-layer graphene in the oil suspension increases in direct proportion to the increase in: the drum radius; the drum rotation speed; the initial concentration of graphite. A parametric row of rod drum mills was developed for the production of graphene oil-based suspensions with a dry graphene productivity from 0.096 to $0.884 \mathrm{~g} / \mathrm{h}$.

\section{References}

1. Bodurov P., Genchev V. New and More Effective Grinding Bodies for Drum Mills. Alternative of the Spherical Grinding Bodies. Journal of Multidisciplinary Engineering Science and Technology (JMEST) 2015, vol. 2, issue 9, September, pp. 2516-2520.

2. Henein H., Brimacobe J. K., Watkison A.P. The Modeling of Transverse Solids Motion in Rotary Kils, Metallurgical Transactions 1983, B 14(2), pp. 207-220.

3. Taberlet N., Richard P., John Hinch E. S shape of a granular pile in a rotating drum. Physical Review E 2006, 73. doi: 10.1103/PhysRevE.73.050301

4. Brewster R., Grest Gary S., Alex J. Levine Effects of cohesion on the surface angle and velocity profiles of granular material in a rotating drum Physical Review E 2009, 79, doi: 10.1103/PhysRevE.79.011305

5. Rogachev A.S., Moskovskikh D.O. Nepapushev A.A., Sviridova T.A., Vadchenko S.G. Experimental investigation of milling regimes in planetary ball mill and their influence on structure and reactivity of gasless powder exothermic mixtures. Powder Technology 2015, vol. 274, pp. 44-52.

6. Santomaso M., Olivi P. Canu Mixing kinetics of granular materials in drums operated in rolling and cataracting regime. Powder Technology 2005, vol. 152, pp. 41-51.

7. Nan Gui, Xingtuan Yang, Jiyuan Tu, Shengyao Jiang Numerical simulation and analysis of mixing of polygonal particles in 2D rotating drums by SIPHPM method. Powder Technology 2017, vol. 318, pp. 248-262.

8. Pershin V.F., Selivanov Yu.T. Modeling of the mixing of particulate materials in continuous-circulating mixers.Teoreticheskie Osnovy Khimicheskoi Tekhnologii. vol. 37, issue 6, 2003, pp. 629-636.

9. Selivanov Yu.T., Pershin V.F. Experimental study of axial mixing in a drum mixer. Theoretical Foundations of Chemical Engineering 2004, vol. 38, issue 1, January, pp. 99-101
10. Selivanov Yu.T., Pershin V.F. Experimental study of longitudinal dispersion in a drum mixer. Teoreticheskie Osnovy Khimicheskoi Tekhnologii, 2004, vol. 38, issue 1, pp. 103-105.

11. Xue B.C., Liu T., Huang H., Liu E.B. The effect of the intimate structure of the solid binder on material viscosity during drum granulation. Powder Technology. 2014, vol. 253 , pp. 584-589.

12. Briens Lauren, Bowden-Green Breanna. A comparison of drum granulation of biochars. Powder Technology. 2019, vol. 43, pp. 723-732.

13. Rafaella F. Rodrigues, Sergio R. Leite, Dyrney A. Santos, Marcos A. S. Barrozo Drum granulation of single super phosphate fertilizer: Effect of process variables and optimization. Powder Technology. 2017, vol. 321, pp. 251-258.

14. Breanna Bowden-Green, Lauren Briens. An investigation of drum granulation of biochar powder. Powder Technology. 2016, vol. 288, pp. 249-254.

15. Tkachev A.G., Kovynev A.A., Nechaev V.M., Pershin V.F. Modeling the screening process. Theoretical Foundations of Chemical Engineering. 2008, vol. 42, issue 4, August, pp. 463-465.

16. Stephane Ngako et.al Numerical investigation of bed depth height, axial velocity and mean residence time of inert particles in steady state industrial cement rotary kiln: Case of Figuil Plant in Cameroon. Powder Technology, 2015, vol. 271, pp. 221-227.

17. Yassine Demagh, Hocine Ben Moussa, Mohammed Lachi, Samira Noui, Lyes Bor Surface particle motions in rotating cylinders: Validation and similarity for an industrial scale kiln. Powder Technology, 2012, vol. 224, pp. 260-272.

18. Santos D.A., Petri I.J., Duarte C.R., Barrozo M.A.S. Experimental and CFD study of the hydrodynamic behavior in a rotating drum. Powder Technology, 2013, vol. 250, pp. 52-62.

19. Delele M.A., Weigler F., Franke G., Mellmann J. Studying the solids and fluid flow behavior in rotary drums based on a multiphase CFD model. Powder Technology, 2016, vol. 292, pp. 260-271.

20. Pershin V.F. Energy method for describing granular motion in a smooth rotating cylinder. Theoretical Foundations of Chemical Engineering, 1988, vol. 22, issue 2, November, pp. 196-201.

21. Shi F., Xie W. A specific energy-based ball mill model: From batch grinding to continuous operation. Miner. Eng. 2016, vol. 86, pp 66-74.

22. Mulenga F.K. Sensitivity analysis of Austin's scaleup model for tumbling ball mills - Part 1. Effects of batch grinding parameters.Powder Technol. 2017, vol. 311, pp. 398-407.

23. Mulenga F.K. Sensitivity analysis of Austin's scaleup model for tumbling ball mills - Part 2. Effects of full-scale milling parameters. Powder Technol. 2017, vol. 317, pp. 6-12.

24. Chimwani N., Mulenga F.K., Hidebrandt D., Glasser D., Bwalya M.M. Scale-up of batch grinding data for simulation of industrial milling of platinum group minerals ore. Miner. Eng. 2014, vol. 63, pp. 100-109.

25. Pershin V.F. Calculation of the distribution of friable material in a smooth rotating drum. Chemical and Petroleum Engineering, 1989, vol. 24, issue 5-6, January, pp. 261-264. 
26. Bilge Öksüzoğlu, Metin Uçurum An experimental study on the ultra-fine grinding of gypsum ore in a dry ball mill. Powder Technology, 2016, vol. 291, pp. 186-192.

27. Deniz V. Influence of interstitial filling on breakage kinetics of gypsum in ball mill. Adv. Powder Technol., 2011, vol. 22 , pp. 512-517.

28 Epstein B. Logarithmic-normal distribution in breakage of solids. Ind. Eng. Chem. 1948, vol. 40, pp. 22892291.

29. Herbst J.A., Fuerstenau D.W. The zero-order production of fine sizes in comminution and its implications in simulation. Trans. AIME. 1968, vol. 241, pp. 538-549.

30. Chenje T.W., Simbi D.J., Navara E. Relationship between microstructure, hardness, impact toughness and wear performance of selected grinding media for mineral ore milling operations. Mater. Des. 2004, vol. 25, pp. 11-18.

31. Ipek H. Effect of grinding media shapes on breakage parameters. Part. Syst. Charact. 2007, vol. 24, pp. 229-235.

32. Kiangi K.K., Michael H.M. Particle filling and size effects on the ball load behaviour and power in a dry pilot mill: experimental study. Powder Technol. 2008, vol. 187, pp. 79-87.

33. Jankovic A., Sinclair S., The shape of product size distributions in stirredmills. Miner. Eng. 2006, vol. 19, pp. $1528-1536$.

34. Strauch D., Belger P., Hofer H., Merz M., PltissStaufer A.G., U.S. Patent 4767464 (1988).

35. Werner R. Effect of extenders with narrow and broad particle size distribution on the properties of coating. Technol. Form. 2000, vol. 72, issue 90.3, pp. 71-76.

36. Nomura S. Dispersion properties for residence time distributions in tumbling ball mills. Powder Technology, 2012, vol. 222, pp. 37-51.

37. Novoselov K.S. et al Electric field effect in atomically thin carbon films. Science, 2004, vol. 306, pp. 666-669.

38. Seol J.H. et al. Two-dimensional phonon transport in supported graphene. Science, 2010, vol. 328, pp. 213-216.

39. Chen S.S. et al. Thermal conductivity of isotopically modified graphene. Nat. Mater., 2012, vol. 11, pp. 203-207.

40. Novoselov K.S. et al. Two-dimensional atomic crystals. P. Natl. Acad. Sci. USA, 2005, vol. 102, pp. 1045110453.

41. Dato A. et al. Substrate-free gas-phase synthesis of graphene sheets. Nano. Lett., 2008, vol. 8, pp. 2012-2016.

42. Kim K.S. et al Large-scale pattern growth of graphene films for stretchable transparent electrodes. Nature, 2009, vol. 457, pp. 706-710.

43. Güler S. H. et al. Production of graphene layer by liquid-phase exfoliation with low sonication power and sonication time from synthesized expanded graphite. Fullerenes Nanotub. Carbon Nanostruct., 2016, vol. 24, pp. 123-127.

44. Güler S. H. Production of grapheme-boron nitride hybrid nanosheets by liquid-phase exfoliation. Optik-Int. J. Light Electron Opt., 2016, vol. 127, pp. 4630-4634.
45. Hernandez Y. et al. High-yield production of graphene by liquid-phase exfoliation of graphite. Nat. Nanotechnol., 2008, vol. 3, pp. 563-568.

46. Berger C. et al. Ultrathin epitaxial graphite: 2D electron gas properties and a route toward graphene-based nanoelectronics. J. Phys. Chem. B., 2004, vol. 108, pp. 19912-19916.

47. Osvath Z. et al. Graphene layers from thermal oxidation of exfoliated graphite plates. Carbon, 2007, vol. 45 , pp. 3022-3026.

48. Güler Hale S., Ömer Güler, Ertan Evin The production of graphene nano layers by using milling exfoliation hybrid process. Received 30 Jun 2016, Accepted 30 Sep 2016, pp. 34-39

49. In-Yup Jeona et al. Edge-carboxylated graphene nanosheets via ball milling PNAS April 10, 2012, vol. 109, issue 15, pp. 5588-5593.

50. Yadav T.P., Yadav R.M., Singh P.D. Mechanical Milling: a Top Down Approach for the Synthesis of Nanomaterials and Nanocomposites. Nanoscience and Nanotechnology 2012, vol. 2, issue 3, pp. 22-48 doi: 10.5923/j.nn.20120203.01

51. Stafford J. et al. Towards Scale-up of Graphene Production via Non-Oxidizing Liquid Exfoliation Methods. AlChE Journal, 2018, vol. 64, issue 9, pp. 3246-3276.

52. Syromyasov A.O., Popov A.I., Pershin V.F. Issledovanie ploskogo dvizheniya odinochnogo sharika vnutri sharovoj mel'nicy [Investigation of the plane motion of a single ball inside a ball mill]. Zhurnal Srednevolzhskogo matematicheskogo obshchestva. 2017, vol. 19, issue 2, pp. 117-125.

53. Zhumagalieva G., Pershin V., Tkachev A., Vorobiev A., Pasko A., Galunin E. Using a Rod Drum Mill for Graphene Masterbatch Production. AIP Conference Proceedings 2018, vol. 2041, 020010; doi: $10.1063 / 1.5079341$

54. Patent No. 168828 RF, U1, MPK B02C 17/00, publ. 21.02.2017. Bul. No. 6. 7 p.

55. Al-Saadi D.A.Y., Pershin V.F., Salimov B.N., Montaev S. A. Modification of graphite greases graphene nanostructures. J. Frict. Wear, 2017, vol. 38, issue 5, pp. 355-358.

56. Pershin V.F., Ovchinnikov K.A., Al-Hilo Z.A.A., Memetov N.R. Tkachev A.G., Galunin E.V. A Graphene Masterbatch for Modification of Frost-Resistant Plastic Lubricants // 6th Intern. Conf. on Production, Energy and Reliability 2018: World Engineering Science \& Technology Congress (ESTCON). doi: 10.1063/1.5079347

57. Pershin V.F., Ovchinnikov K.A., Al-Hilo Z.A.A., Stolyarov R.A., Memetov N.R. Development of environmentally safe lubricants modified by graphene. Nanotechnol. Russ., 2018, vol. 13, issue 5-6, pp. 340-344.

58. Patent № 2670495 RF, B02C 17/10 (2006.01), publ. 23.10.2018. Bul. No. 30. 5 p.

59.Melezhyk A V et al. Optical Properties and Aggregation of Graphene Nanoplatelets. Journal of Nanoscience and Nanotechnology 2016, vol. 16, issue 1, pp. 1067-1075. 